\title{
Silmitasertib Sodium
}

National Cancer Institute

\section{Source}

National Cancer Institute. Silmitasertib Sodium. NCI Thesaurus. Code C85460.

The sodium salt of silmitasertib, an orally bioavailable small-molecule inhibitor of CK2 with potential antineoplastic activity. Silmitasertib selectively binds to and inhibits the enzyme casein kinase II (CK2), which may lead to an inhibition of cellular proliferation. CK2, a protein kinase often overexpressed in a variety of cancer cell types, appears to be correlated with malignant transformation, tumor growth and survival. CK2 regulates a diverse array of pro-survival cellular processes including epidermal growth factor receptor (EGFR) signaling, PI3K/AKT/mT OR signaling, hedgehog $(\mathrm{Hh})$ signaling, Hsp90 machinery, hypoxia, and interleukin (IL)-6 expression. CK2 also regulates the activity of XRCC1 and MDC1, two mediator/adaptor proteins that are essential for DNA repair. 